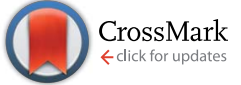

Cite this: J. Mater. Chem. A, 2016, 4, 12648

Received 22nd April 2016 Accepted 20th July 2016

DOI: $10.1039 / \mathrm{c} 6 \mathrm{ta0} 3376 \mathrm{~h}$

www.rsc.org/MaterialsA

\section{An assessment of silver copper sulfides for photovoltaic applications: theoretical and experimental insights $\uparrow$}

\begin{abstract}
Christopher N. Savory, ${ }^{a}$ Alex M. Ganose, ${ }^{\text {ab }}$ Will Travis, ${ }^{c}$ Ria S. Atri, ${ }^{c}$ Robert G. Palgrave and David O. Scanlon ${ }^{\star a b}$

As the worldwide demand for energy increases, low-cost solar cells are being looked to as a solution for the future. To attain this, non-toxic earth-abundant materials are crucial, however cell efficiencies for current materials are limited in many cases. In this article, we examine the two silver copper sulfides AgCuS and $\mathrm{Ag}_{3} \mathrm{CuS}_{2}$ as possible solar absorbers using hybrid density functional theory, diffuse reflectance spectroscopy, XPS and Hall effect measurements. We show that both compounds demonstrate promising electronic structures and band gaps for high theoretical efficiency solar cells, based on Shockley-Queisser limits. Detailed analysis of their optical properties, however, indicates that only AgCuS should be of interest for PV applications, with a high theoretical efficiency. From this, we also calculate the band alignment of AgCuS against various buffer layers to aid in future device construction.
\end{abstract}

\section{Introduction}

The photovoltaic (PV) industry has grown rapidly in the past decade to meet an ever-rising demand for energy that avoids dependence on fossil fuel technology; the importance of such technology is demonstrated by the production of PV devices, which has increased by $40 \%$ each year from 2000 to $2012 .{ }^{1}$ To meet this demand it is crucial that the materials used in these devices are as efficient and cost effective as possible in order to ensure widespread availability. The current material of choice for photovoltaics is crystalline silicon, which benefits from being abundant and having been optimized over the course of half a century to high efficiencies; ${ }^{2}$ however, it also suffers from high energy consumption in the growth of silicon boules, causing a relatively long energetic payback time, ${ }^{3}$ its inherent inefficiency due to its indirect fundamental band gap, and silicon wafers requiring significant thickness $(>100 \mu \mathrm{m})$ in order to absorb sufficient light, increasing material consumption.

Thin-film materials such as CdTe, GaAs and $\mathrm{Cu}(\mathrm{In}, \mathrm{Ga}) \mathrm{Se}_{2}$ (CIGS) have seen an increase in market share within the last decade as cell efficiencies have increased with optimization: up to $21 \%$ for CdTe and CIGS, and $28.8 \%$ for GaAs. ${ }^{4}$ In all these cases, they possess direct band gaps, leading to higher

${ }^{a}$ University College London, Kathleen Lonsdale Materials Chemistry, Department of Chemistry, 20 Gordon Street, London WC1H OAJ, UK. E-mail: d.scanlon@ucl.ac.uk

${ }^{b}$ Diamond Light Source Ltd., Diamond House, Harwell Science and Innovation Campus, Didcot, Oxfordshire OX11 ODE, UK

${ }^{`}$ University College London, Department of Chemistry, London WC1H OAJ, UK

$\dagger$ Electronic supplementary information (ESI) available. See DOI: $10.1039 / \mathrm{c} 6 \mathrm{ta} 03376 \mathrm{~h}$ absorption and allowing for much thinner layers of material for the same cell efficiency as Si. They also boast a much lower energetic cost than silicon, ${ }^{3}$ together with band gaps closer to the optimal theoretical limit for a single junction cell predicted by Shockley and Queisser ${ }^{5}$ - the highest power conversion efficiencies for such a cell are possible between 1.0 and $1.7 \mathrm{eV}$ and a maximum around $1.3 \mathrm{eV}$. Thin film absorbers do have their own problems however, with CIGS restricted by the low relative abundances of indium and gallium, and cadmium and arsenic's toxicity presenting a significant barrier to worldwide application of such technologies.

As such, there has been a recent drive to develop earthabundant, non-toxic alternative photovoltaic materials, such as the antimony and bismuth copper chalcogenides, $\mathrm{Cu}_{3} \mathrm{BiS}_{3}$, $\mathrm{CuSbS}_{2}$ and $\mathrm{CuSbSe}_{2},{ }^{6-9}$ the zinc tin pnictides, $\mathrm{ZnSnN}_{2}$ and $\mathrm{ZnSnP}{ }_{2},{ }^{10-12}$ and the binary antimony chalcogenides, $\mathrm{Sb}_{2} \mathrm{~S}_{3}$ and $\mathrm{Sb}_{2} \mathrm{Se}_{3},{ }^{13,14}$ despite having been shown to have suitable band gaps within the optimal 1.0-1.7 eV range, experimental cell efficiencies for these materials remain low. ${ }^{15-18}$ The current leader in this field is $\mathrm{Cu}_{2} \mathrm{ZnSn}(\mathrm{S}, \mathrm{Se})_{4}$ (CZTSSe) which has a record cell efficiency of $12.6 \%$, a tunable band gap of 1.0-1.5 eV, and is solution processable, ${ }^{19-21}$ however, cell efficiencies have since plateaued with few major advances in efficiency since 2013 as CZTS cells have been limited by a large deficit in open-circuit voltage compared to the band gap. ${ }^{22-24}$ The kesterite system also has complex defect physics due to its quaternary nature, meaning close control of $\mathrm{Cu}$ and $\mathrm{Zn}$ proportions, as well as inclusion of $\mathrm{Na}$, is often critical for suppressing non-radiative recombination and attaining high efficiency cells. ${ }^{25-30}$ 
As a result, we look towards the simpler ternary silver copper sulfides, $\mathrm{AgCuS}$ (stromeyerite) and $\mathrm{Ag}_{3} \mathrm{CuS}_{2}$ (jalpaite). The silver copper sulfides have been examined historically for their ionic conductivity at high temperatures, and a number of studies have examined their phase behaviour. ${ }^{\mathbf{3 1}, 32}$ The most recent structural studies on the room temperature phases of these two compounds have been performed by Baker et al. ${ }^{33,34}$ and Trots et $a .^{35,36}$ using single crystal X-ray and neutron powder diffraction. Previous theoretical investigations of AgCuS have focused on its high temperature cubic phase ${ }^{37}$ and its behaviour under pressure; ${ }^{38}$ the most recent work, however, showed that it exhibits p-type conductivity with a significant thermopower $\left(\sim 665 \mu \mathrm{V} \mathrm{K}^{-1}\right)$ at room temperature. ${ }^{39} \mathrm{Ag}_{3} \mathrm{CuS}_{2}$ has previously, in combination with $\mathrm{Ag}_{2} \mathrm{~S}$ and $\mathrm{Ag}$, been shown to exhibit photocatalytic behaviour, ${ }^{40}$ but it is of immediate interest due to the recent publication of two solar cells utilizing it as an absorber layer. ${ }^{41,42}$

In this article, we examine the silver copper sulfides using hybrid density functional theory (DFT) with an aim to critically assess their suitability as photovoltaic absorber materials. Two different structures of the silver copper sulfides were investigated using DFT: the room temperature, or $\beta, C m c 2_{1}$ phase of $\mathrm{AgCuS},{ }^{33}$ and the room temperature $\mathrm{I}_{1} /$ amd phase of $\mathrm{Ag}_{3} \mathrm{CuS}_{2}{ }^{36}$ These structures are depicted in Fig. 1. Both of the structures studied are connected in all 3 dimensions: RT-AgCuS consists of zig-zag Ag-S chains, with the silver atoms linearly coordinated, which are bridged along $\mathrm{a}$ and $\mathrm{b}$ by 3-coordinate copper atoms. RT- $\mathrm{Ag}_{3} \mathrm{CuS}_{2}$ contains two different silver environments - octahedrally coordinated, and highly distorted tetrahedrally coordinated to sulfur; these environments are face-sharing and create the ' $X$ ' shaped structure seen in the $\{011\}$ plane in Fig. $1 b$, while the copper atoms are linearly coordinated and bridge the channels in the structure. Different copper coordination environments have previously been observed to impact upon electronic properties in semiconductors, so the differences between these two structures are of interest. ${ }^{43}$ These structures were optimized computationally using a hybrid exchange-correlation functional, and the relative accuracy compared to experiment was assessed. From this, we present a thorough exploration of the optical and electronic properties of the systems of interest, with attention to how these may affect their photovoltaic behaviour.

\section{Methods}

\subsection{Theoretical}

Each structure in this report was optimized and electronic structure calculated using periodic DFT using the Vienna Ab Initio Simulation Package (VASP), which implements all the DFT and hybrid DFT functionals mentioned in this report. ${ }^{44-47}$ The primary functional used was the hybrid functional HSE06. ${ }^{48}$ This incorporates 25\% Hartree Fock exchange in addition to 75\% exchange from the Generalised Gradient Approximation (GGA) functional, $\mathrm{PBE}^{\mathbf{4 9}}$ additionally in HSE06, the HF exchange is screened using a parameter of $\omega=0.11 \mathrm{bohr}^{-1}$ such that it is only significant at short range. The projectoraugmented wave method was used to account for valence and core electron interactions. ${ }^{50}$ A cutoff energy of $350 \mathrm{eV}$, a $k$-mesh spacing of $0.04 \AA^{-1}$ along each reciprocal vector and a convergence criterion of $0.01 \AA^{-1}$ on the forces per atom were used in all calculations. By utilising a hybrid functional, we hope to avoid the well-known problem with GGA-based DFT methods: that they can severely underestimate semiconductor band gaps. ${ }^{51}$ Also, we might expect that hybrid functionals, by including correct Hartree-Fock electron exchange, will also avoid some of the self-interaction error inherent in DFT calculations, which becomes particularly significant in systems which contain strongly correlated d electrons, like the silver copper sulfides. HSE06 was chosen as it has been shown to give accurate measurements of semiconductor band gaps in a)

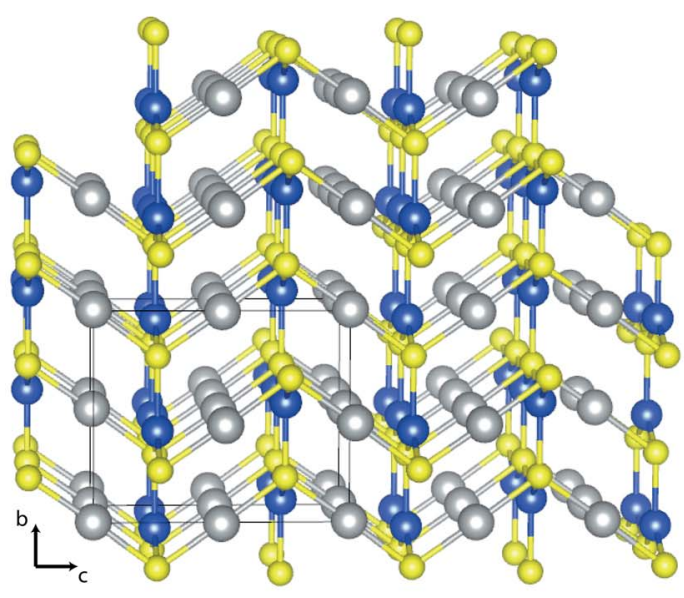

b)

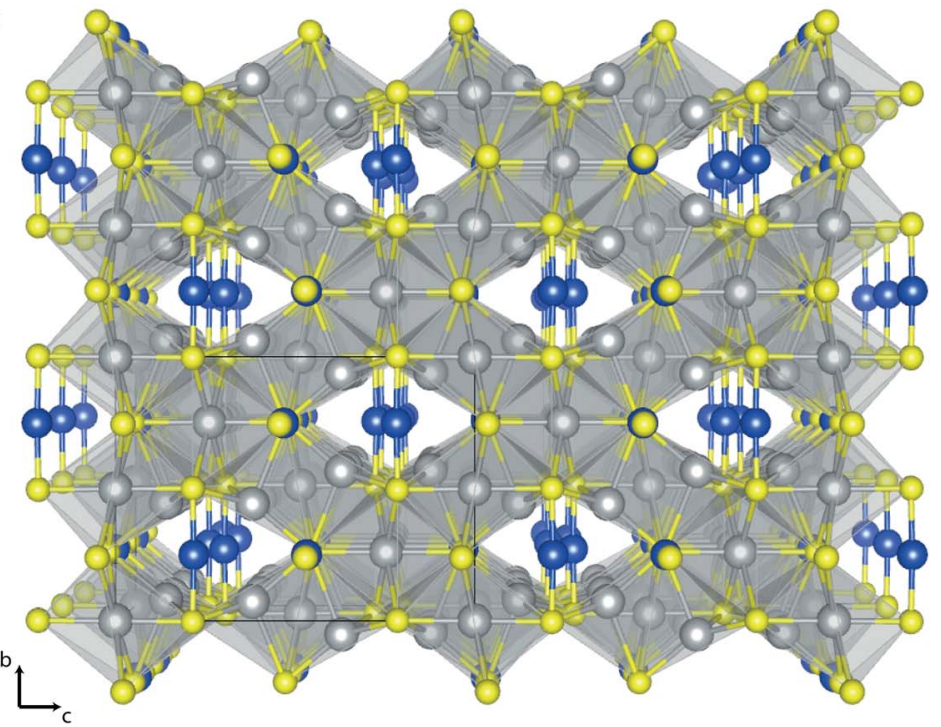

Fig. 1 Crystal structures of (a) RT-AgCuS and (b) RT-Ag ${ }_{3} \mathrm{CuS}_{2}$. A single unit cell is marked in each, and the following atom labels are used: Ag atoms in grey, $\mathrm{Cu}$ in blue and $\mathrm{S}$ in yellow. 
comparison to experiment in work on other semiconductor systems. ${ }^{\mathbf{8} 52-55}$ To calculate the optical properties of the system, the method developed by Furthmüller et $a{ }^{.56}$ was used to calculate the high-frequency dielectric function, from which the absorption coefficient can be derived. The valence band alignment of $\mathrm{AgCuS}$ was performed using the core-level alignment approach developed by Wei and Zunger. ${ }^{57}$ All crystal structures in this report were drawn in the VESTA visualisation program. ${ }^{\mathbf{5 8}}$

\subsection{Experimental section}

Synthesis of AgCuS. AgCuS was synthesised using the hydrothermal method proposed by Tokuhara et al. ${ }^{59}$ Non-stoichiometric quantities of Ag (1.4314 g, $0.0133 \mathrm{~mol}), \mathrm{Cu}(1.0306 \mathrm{~g}$, $0.0162 \mathrm{~mol})$, and $\mathrm{S}(0.4727 \mathrm{~g}, 0.0147 \mathrm{~mol})$, using a molar ratio of $0.9: 1.1: 1.0$, were ground together in an agate pestle and mortar. Of this mixture $0.5 \mathrm{~g}$ was transferred to a Teflon-lined steel autoclave $(45 \mathrm{~mL})$ together with $15 \mathrm{~mL}$ distilled water. The reaction vessel was oven heated at $180{ }^{\circ} \mathrm{C}$ for $10 \mathrm{~h}$, before being cooled slowly to room temperature. The AgCuS was isolated in quantitative yield via filtration; it was washed several times with distilled water and dried.

Synthesis of $\mathbf{A g}_{3} \mathrm{CuS}_{2} . \mathrm{Ag}_{3} \mathrm{CuS}_{2}$ was synthesised from a nonstoichiometric molar ratio mixture of $3.05: 1.00: 2.00$ of elemental Ag (1.6039 g, $0.0143 \mathrm{~mol}), \mathrm{Cu}(0.3000 \mathrm{~g}, 0.0047 \mathrm{~mol})$ and $\mathrm{S}(0.3027 \mathrm{~g}, 0.0094 \mathrm{~mol})$. The reagents were ground together in an agate pestle and mortar and added to a $1 \mathrm{~cm}$ diameter quartz tube. The tube was evacuated and flame sealed before heating at $5{ }^{\circ} \mathrm{C} \min ^{-1}$ to $500{ }^{\circ} \mathrm{C}$ with a dwell time of $10 \mathrm{~h}$ followed by cooling slowly to room temperature. $\mathrm{Ag}_{3} \mathrm{CuS}_{2}$ was obtained in good yield without further washing or purification.

Characterisation. Powder X-ray diffraction (PXRD) data was collected on a Stoe StadiP diffractometer using $\mathrm{Cu} \mathrm{K} \alpha 1$ ( $\lambda=$ $1.54056 \AA$ A) radiation. $0.5 \mathrm{~mm}$ capillaries were filled with powdered samples and data were collected over the $2 \theta$ range 5$60^{\circ}$ in steps of $0.5^{\circ}$ at $20 \mathrm{~s}$ per step. Optical diffuse-reflectance data was recorded between 300 and $2000 \mathrm{~nm}$, with a data collection step of $1 \mathrm{~nm}$, using a Lambda 950 spectrophotometer equipped with an integrating sphere at ambient temperature. $\mathrm{AgCuS}$ and the $\mathrm{Ag}_{3} \mathrm{CuS}_{2}$ were pressed into $13 \mathrm{~mm}$ diameter pellets at 5 bar with thicknesses of $1.04 \mathrm{~mm}$ and $1.24 \mathrm{~mm}$ for $\mathrm{AgCuS}$ and $\mathrm{Ag}_{3} \mathrm{CuS}_{2}$ respectively. The Hall coefficient, electrical resistivity, carrier concentration and carrier mobility of the AgCuS pellet were measured using van der Pauw geometry on a Ecopia Hall Measurement System (HMS-3000) at room temperature using four silver paint contacts. Four point probe measurements were performed, giving sheet resistance for $\mathrm{Ag}_{3} \mathrm{CuS}_{2}$, as resistance was too high for Hall effect measurement. X-ray photoemission spectroscopy (XPS) measurements were recorded using a Thermo Scientific Al-K $\alpha$.

\section{Results and discussion}

\subsection{AgCuS}

Firstly, AgCuS was considered: calculated lattice parameters of $a=4.042 \AA, b=6.752 \AA$ and $c=8.431 \AA$ were obtained; a comparison of this geometry optimization with experiment is

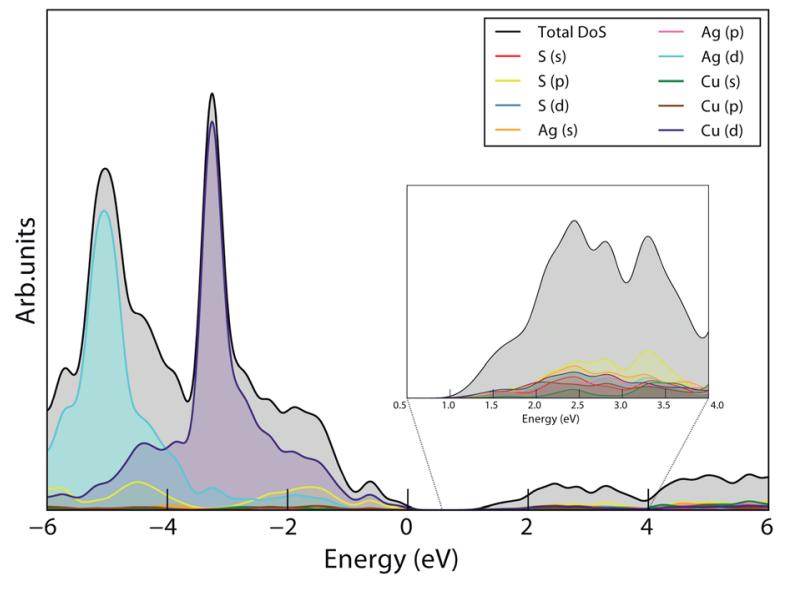

Fig. 2 Total and partial density of states (DoS) of AgCuS, with HSE06; individual partial DoS are labelled in legend, valence band maximum (VBM) set to $0 \mathrm{eV}$.

shown in the ESI (ST1†). The total and partial Density of States (DoS) diagram is shown in Fig. 2, demonstrating that the valence band is primarily composed of $\mathrm{Ag} \mathrm{d}, \mathrm{Cu} d$ and $\mathrm{S}$ p states, while $\mathrm{Cu} d$ and $\mathrm{S}$ p states also dominate the conduction band minimum, with some Ag s contribution. The additional localisation of the strongly correlated $\mathrm{Cu} d$ and $\mathrm{Ag} d$ states in HSE06 due to its partial correction of self-interaction error causes the bulk of these to be low in energy and leading to a large proportion of $\mathrm{S}$ p states at the valence band edge. A similar shift is seen in other $\mathrm{Cu}(\mathrm{I})$ and $\mathrm{Ag}$ systems. ${ }^{60,61}$

The HSE06 electronic band structure of AgCuS is shown in Fig. 3. The HSE06 band gap is direct, with a predicted value of $1.27 \mathrm{eV}$, well within the ideal range for photovoltaics, and corresponds to a maximum theoretical efficiency of around 33\% under AM 1.5 illumination in the Shockley-Queisser (SQ) limit. Comparing to experimental work done by Guin et al. ${ }^{39}$ which found an approximate band gap of $0.9 \mathrm{eV}$ using optical diffuse reflectance measurements, this HSE06 result appears to be an overestimation. The band structure shows very significant dispersion in the valence band, and also a similar degree of dispersion in the conduction band. The resultant effective masses have been calculated from the HSE06 band structure and are listed in Table 1, showing that there is some anisotropy in the valence band effective masses, that they are particularly low $\left(<0.4 m_{0}\right.$, indicating the possibility of high mobility $\left.{ }^{62}\right)$ along the copper-sulfur layers in the (001) plane, and they are close to those predicted in the kesterites, like CZTS. ${ }^{63}$ The electron effective mass is on average higher than that of the holes which, while unusual, has been seen in other photoabsorbers such as methylammonium lead iodide and BiSI. ${ }^{54,64}$ The magnitudes of $m_{\mathrm{e}}$ are also similar to those of other promising Cu-based photovoltaic absorbers. ${ }^{6}$

\section{2 $\quad \mathrm{Ag}_{3} \mathrm{CuS}_{2}$}

The results from the structural optimizations of the $I 4_{1} /$ amd phase of $\mathrm{Ag}_{3} \mathrm{CuS}_{2}$ are displayed in ESI Table 2, $\dagger$ with the calculated lattice parameters of $a=8.835 \AA$ and $c=11.801 \AA$. 


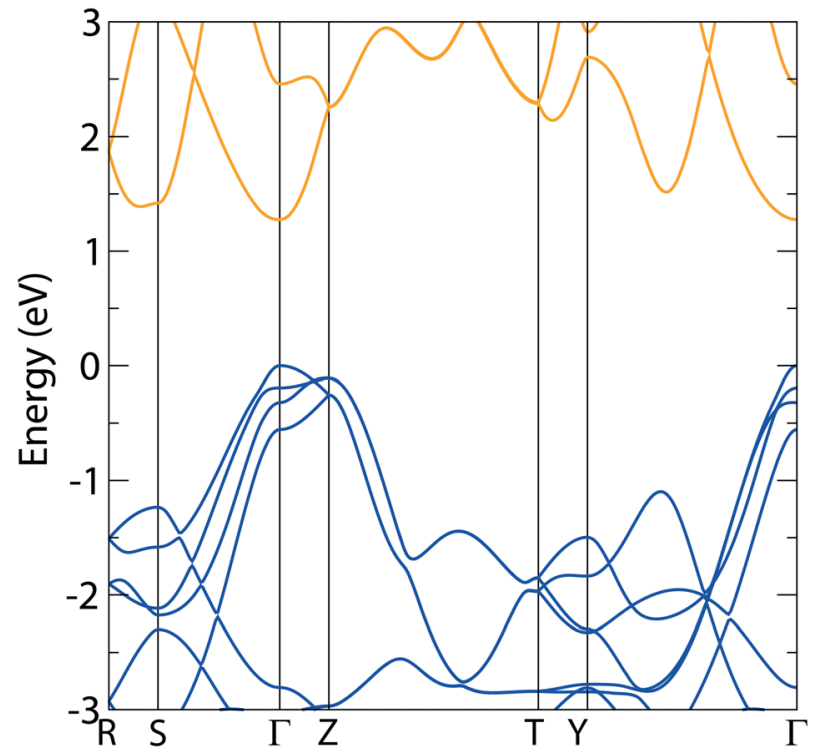

Fig. 3 Band structure diagram of AgCuS using the HSEO6 functional, showing a direct $1.27 \mathrm{eV}$ band gap; valence band marked in blue, conduction band marked in orange, VBM set to $0 \mathrm{eV}$.

Table 1 Calculated effective masses of AgCuS and /4 $1 /$ amd $\mathrm{Ag}_{3} \mathrm{CuS}_{2}$ from HSEO6 band structures

\begin{tabular}{lllllll}
\hline & \multicolumn{3}{c}{ Valence band $\left(m_{0}\right)$} & \multicolumn{3}{c}{ Conduction band $\left(m_{0}\right)$} \\
\hline AgCuS & $\Gamma \rightarrow \mathrm{S}$ & $\Gamma \rightarrow \mathrm{Y}$ & $\Gamma \rightarrow \mathrm{Z}$ & $\Gamma \rightarrow \mathrm{S}$ & $\Gamma \rightarrow \mathrm{Y}$ & $\Gamma \rightarrow \mathrm{Z}$ \\
& 0.311 & 0.242 & 1.795 & 1.362 & 0.736 & 0.493 \\
\hline \multicolumn{3}{c}{ Valence band $\left(m_{0}\right)$} & & Conduction band $\left(m_{0}\right)$ \\
\hline $\mathrm{Ag}_{3} \mathrm{CuS}_{2}$ & $\Gamma \rightarrow \mathrm{N}$ & $\Gamma \rightarrow \mathrm{X}$ & $\Gamma \rightarrow \mathrm{Z}$ & $\Gamma \rightarrow \mathrm{N}$ & $\Gamma \rightarrow \mathrm{X}$ & $\Gamma \rightarrow \mathrm{Z}$ \\
& 0.522 & 0.598 & 0.279 & 0.281 & 0.322 & 0.206
\end{tabular}

The density of states diagram for $\mathrm{Ag}_{3} \mathrm{CuS}_{2}$ shows similar trends to those seen in AgCuS: the same states make up the conduction and valence bands, albeit with a greater concentration of $\mathrm{Ag} \mathrm{d}$ states due to the stoichiometry of the system, and localization of the $\mathrm{Ag}$ and $\mathrm{Cu}$ valence states within the valence band, resulting in a high DOS there - the density of state diagram for $\mathrm{Ag}_{3} \mathrm{CuS}_{2}$ is enclosed in the ESI (Fig. SF2 $\dagger$ ). At the valence band maximum, unlike AgCuS, the Ag d states dominate in proportion over the $\mathrm{Cu} \mathrm{d}$, although the bulk of the $\mathrm{Ag}$ d states remain lower in energy. The HSE06 band structure is displayed in Fig. 4. The most significant result from this is the direct fundamental band gap of $1.05 \mathrm{eV}$, which is encouraging, as it corresponds to a SQ limit of around $30 \%$, well within the suitable range for $\mathrm{PV}$, and is consistent with $\mathrm{Ag}_{3} \mathrm{CuS}_{2}$ 's observed photoactivity. ${ }^{41}$ The band structure also demonstrates good dispersion in both the conduction and valence band, similar to that seen in $\mathrm{AgCuS}$, indicating significant electron and hole mobility through the 3D structure. The effective masses for electrons and holes were calculated from the band structure, and are shown in Table 1 ; with average values of $0.270 m_{0}$ and

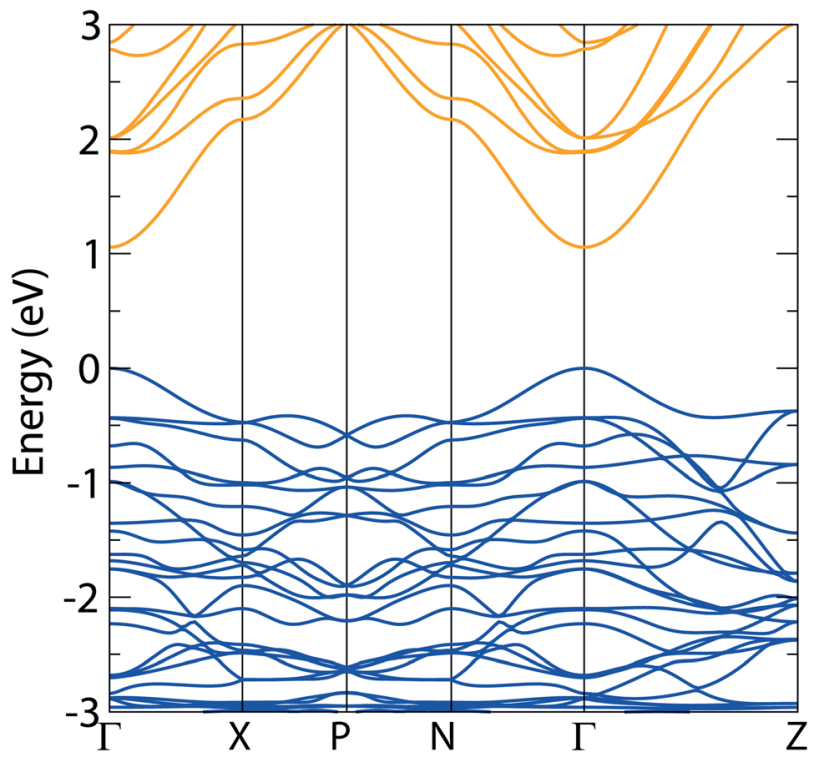

Fig. 4 Band structure diagrams of $14_{1} /$ amd $\mathrm{Ag}_{3} \mathrm{CuS}_{2}$ using HSE06, demonstrating a direct $1.05 \mathrm{eV}$ band gap; valence band marked in blue, conduction band marked in orange, VBM set to $0 \mathrm{eV}$.

$0.466 m_{0}$ respectively, these are comparable to other photovoltaic materials demonstrating high carrier mobilities. ${ }^{\mathbf{6 5 , 6 6}}$

\subsection{Synthesis and experimental analysis}

In addition to this theoretical work, experimental work was carried out to synthesise and characterise both compounds, and to verify some of the theoretical predictions above. Both compounds were synthesised from a mixture of their constituent elements: AgCuS was obtained using a hydrothermal method similar to that used by Tokuhara et al. ${ }^{59}$ while $\mathrm{Ag}_{3} \mathrm{CuS}_{2}$ used a typical high temperature synthesis from the elements. In both cases, the products were obtained as black powders, and identified using powder X-ray diffraction; the resulting patterns were indexed in previously obtained space groups of $C m c 2_{1}$ for AgCuS and $14_{1} /$ amd for $\mathrm{Ag}_{3} \mathrm{CuS}_{2},{ }^{33,34}$ and are shown in comparison to patterns simulated from those previous structures in ESI Fig. SF5. $\dagger$ Lattice parameters obtained by least squares refinement of the powder XRD peak positions were $a=4.0623(1) \AA$, $b=6.6254(2) \AA, c=7.9692(2) \AA$ for AgCuS, and $a=8.6370(2) \AA$, $c=11.7688(5) \AA$ for $\mathrm{Ag}_{3} \mathrm{CuS}_{2}$. A good match between the simulated and experimental patterns (no more than $0.2 \%$ difference in any lattice parameter), with a lack of impurity peaks, indicates that the powders obtained were single-phase and sufficiently pure for further analysis. Williamson Hall plots were used to estimate the volume weighted mean crystallite size. For AgCuS, the mean size was $50 \mathrm{~nm}$, while for $\mathrm{Ag}_{3} \mathrm{CuS}_{2}$ the mean size was $70 \mathrm{~nm}$; the larger size is consistent with the high temperature synthesis route used. Optical reflectance measurements were then performed on the samples to assess the experimental band gap; the resultant Kubelka-Munk plots are shown in Fig. 5. The experimental optical band gaps observed, 1.25 and $1.05 \mathrm{eV}$ for $\mathrm{AgCuS}$ and $\mathrm{Ag}_{3} \mathrm{CuS}_{2}$ respectively, correlate very well with our predicted direct fundamental band 

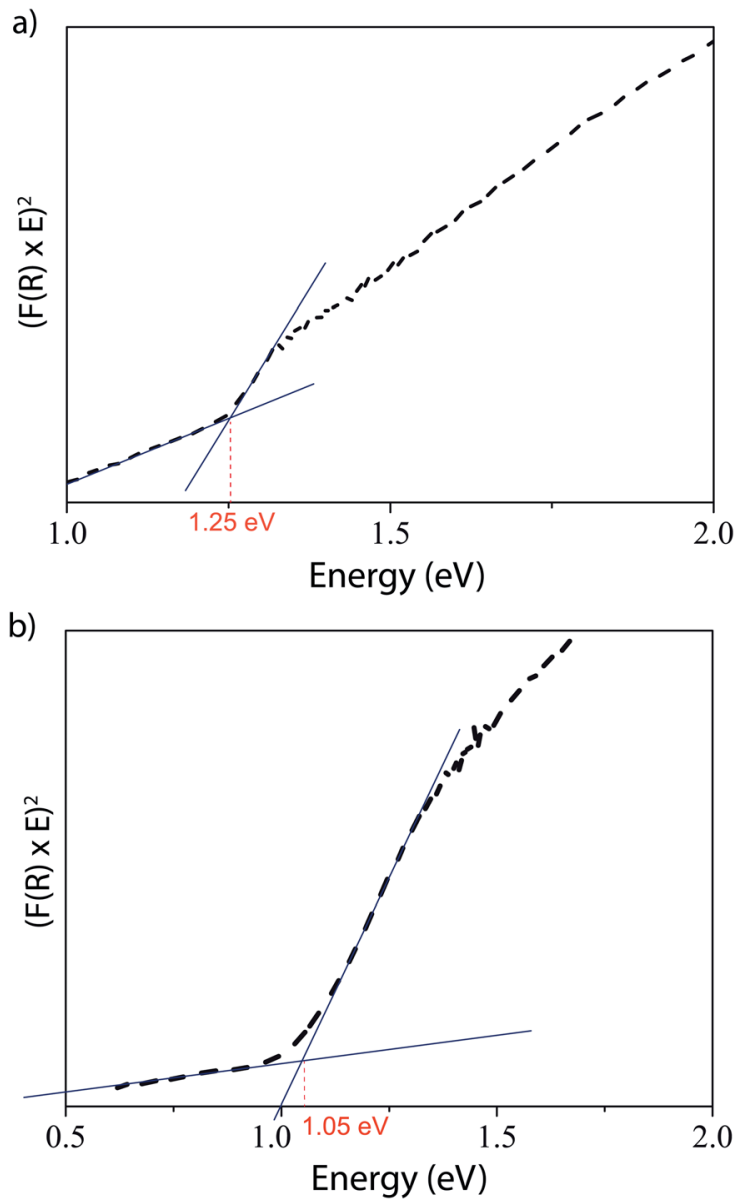

Fig. 5 Kubelka-Munk plot from diffuse reflectance measurement of (a) AgCuS and (b) $\mathrm{Ag}_{3} \mathrm{CuS}_{2}$. Intersections of background and absorption marked, giving the optical band gaps, in red.

gaps of 1.27 and $1.05 \mathrm{eV}$ from the HSE06 calculations. A range of optical band gaps have been reported for AgCuS from 0.9-1.2 eV (ref. 39 and 67) with larger band gaps associated with small particle sizes. Our band gap value is at the larger end of this range, which is more in-keeping with our calculated value.

X-ray photoelectron spectroscopy (XPS) was also carried out on both materials. Core-level XPS, which demonstrates that Ag, $\mathrm{Cu}$ and $\mathrm{S}$ are present in both samples with no impurity states, is included in ESI Fig. SF6 and SF7. $\dagger$ As presented, both AgCuS and $\mathrm{Ag}_{3} \mathrm{CuS}_{2}$ samples gave XPS survey spectra indicating the presence of $\mathrm{Cu}, \mathrm{Ag}, \mathrm{S}$ and $\mathrm{O}$, as well as adventitious carbon on the surface. In both cases etching with $2 \mathrm{keV} \mathrm{Ar}^{+}$ions for $100 \mathrm{~s}$ was sufficient to totally remove the oxygen to below the detection limit of the instrument ( $c a .0 .5$ atomic\%). After etching the measured surface composition of the AgCuS sample was $\mathrm{Ag}_{0.93} \mathrm{CuS}_{0.96}$ and that of the $\mathrm{Ag}_{3} \mathrm{CuS}_{2}$ sample was $\mathrm{Ag}_{3.05} \mathrm{Cu}_{1.03} \mathrm{~S}_{2.01}$. Given the usually quoted XPS composition error of up to $10 \%$, these compositions are consistent with the expected formulae. The high resolution spectra discussed below all refer to the etched samples.

For both $\mathrm{AgCuS}$ and $\mathrm{Ag}_{3} \mathrm{CuS}_{2}$, the $\mathrm{Ag} 3 \mathrm{~d}$ high-resolution XPS scans in (a) of both Fig. S6 and $\mathrm{S} 7 \dagger$ show the expected spin orbit doublet with symmetrical peaks, the $\mathrm{Ag} 3 \mathrm{~d}_{5 / 2}$ appearing at 368.2
$\mathrm{eV}$ in both compounds. These values are consistent with data reported by Chowdari et al. for $\mathrm{Ag}_{2} \mathrm{~S},{ }^{68}$ and together with the absence of loss features which would be observed at the high binding energy side of the core line peaks if $\mathrm{Ag}$ metal were present indicate that $\mathrm{Ag}^{+}$is the only detectable $\mathrm{Ag}$ species by XPS. Fig. $\mathrm{S} 6$ and $\mathrm{S} 7 \dagger$ (b) show the $\mathrm{Cu} 2 \mathrm{p}$ high resolution scans for $\mathrm{AgCuS}$ and $\mathrm{Ag}_{3} \mathrm{CuS}_{2}$, with $\mathrm{Cu} 2 \mathrm{p}_{3 / 2}$ peaks at $932.5 \mathrm{eV}(\mathrm{AgCuS})$ and $932.7 \mathrm{eV}\left(\mathrm{Ag}_{3} \mathrm{CuS}_{2}\right)$ corresponding to known values for $\mathrm{Cu}_{2} \mathrm{~S}^{69,70}$ The $\mathrm{Cu} 2 \mathrm{p}$ peak shape is highly sensitive to $\mathrm{Cu}$ oxidation state, with $\mathrm{Cu}_{2}^{+}$states displaying strong satellite features, and $\mathrm{Cu}$ metal showing asymmetry due to plasmon energy loss processes. Therefore the symmetric $\mathrm{Cu} 2 \mathrm{p}$ peaks observed here, and the absence of any satellite peaks, indicates that $\mathrm{Cu}^{+}$is the only copper oxidation state detected. The spin orbit components of the $\mathrm{S} 2 \mathrm{p}$ doublet are resolved for both compounds ((c) in Fig. S6 and $S 7 \dagger)$, with the $S 2 p_{3 / 2}$ peak measured at a binding energy of $161.5 \mathrm{eV}$ in each case, consistent with expectations for $\mathrm{S}^{2-}$ ions. ${ }^{69}$ After etching, no higher binding energy $S 2$ p peaks, corresponding to sulfate species, were observed, indicating that any oxidation is limited to the surface.

Additionally, the XPS at the valence band edge was also recorded and compared to the calculated density of states diagrams, shown in Fig. 6 . In both cases, the DoS is scaled using
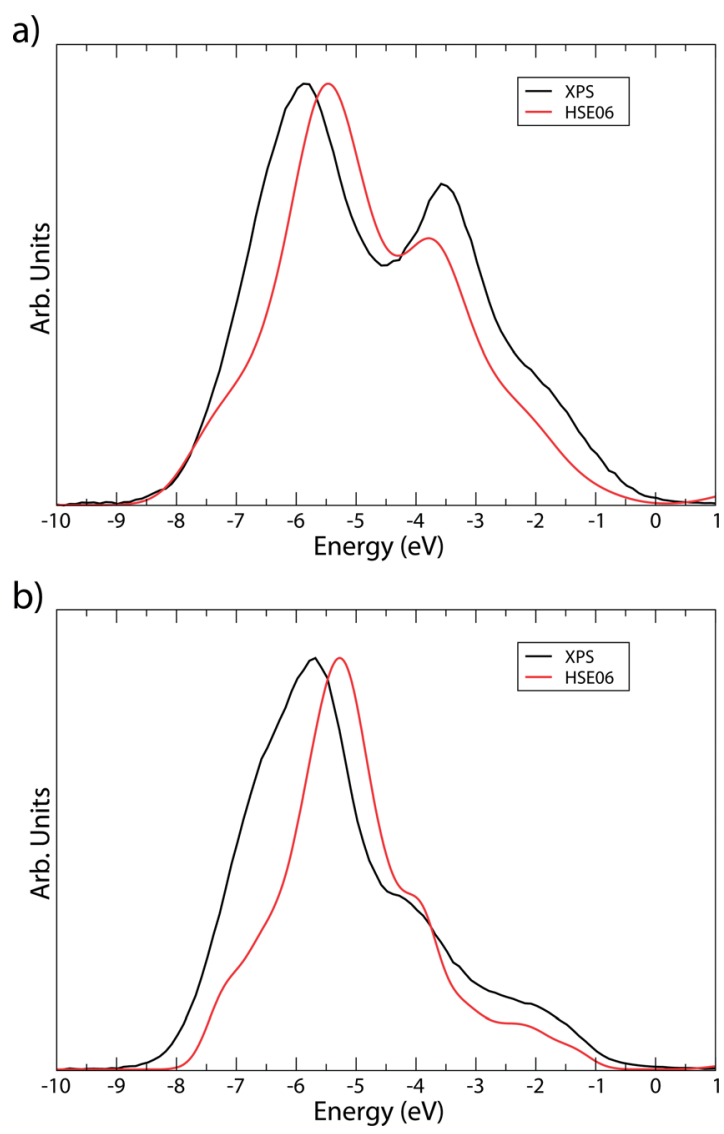

Fig. 6 Comparison of valence band XPS (black) and HSEO6 density of states (red) for (a) AgCuS and (b) $\mathrm{Ag}_{3} \mathrm{CuS}_{2}$. Valence band edge has been normalized to $0 \mathrm{eV}$. 
atomic orbital photoionisation cross-sections ${ }^{71}$ and the Gaussian smearing is adjusted to simulate experimental broadening. The major features of each XPS are matched well in the DoS, further indicating that the HSE06 functional predicts even the highly-correlated $\mathrm{Cu}$ and $\mathrm{Ag}$ states successfully.

\subsection{Optical properties}

As noted above, strong optical absorption is also necessary for good photovoltaic performance and so to further assess the suitability of these compounds, the attenuation coefficient, $\alpha$, of both materials was calculated through the dielectric tensor. ${ }^{56}$ The resultant plot of $\alpha$ against energy is plotted in Fig. 7. These results show that the predicted optical gaps, given by $(\alpha h \nu)^{2}$, are $\sim 0.2 \mathrm{eV}$ above the fundamental gaps for both materials. It should be remembered that our calculations are performed athermally, and we anticipate that the effects of temperature in the experimental measurements will lead to some lattice expansion and resultant shrinkage of the band gap, hence the fortuitous agreement between fundamental calculated and experimental optical band gaps in this report. Another source for this discrepancy may be the presence of defects, which were not accounted for in the optical calculations. Strong absorption, characterized by $\alpha>10^{4}$, from both materials is also above that of the fundamental band gap - this is due to the VBM-CBM transition being symmetry forbidden in both cases. As a result, the lowest direct transition for $\mathrm{AgCuS}$ is increased to $1.46 \mathrm{eV}$, which is still within the suitable range for photovoltaic applications. This transition is marked in Fig. SF8 in the ESI, $\dagger$ originating from the band below the VBM, which is similarly dominated by $\mathrm{Cu} \mathrm{d}$ and $\mathrm{S}$ p orbitals, with very little $\mathrm{Ag}$ contribution. On the other hand, $\mathrm{Ag}_{3} \mathrm{CuS}_{2}$ has multiple symmetry disallowed transitions, with the lowest direct allowed transition at $2.01 \mathrm{eV}$, indicating it could be a significantly poorer candidate for devices. This is reflected in the predicted absorption coefficient: for AgCuS, $\alpha$ increases relatively smoothly from $\sim 0.3 \mathrm{eV}$ above the band gap, while the absorption for $\mathrm{Ag}_{3} \mathrm{CuS}_{2}$ remains low $\left(<10^{4} \mathrm{~cm}^{-1}\right)$ until above $2 \mathrm{eV}$. The target band for the

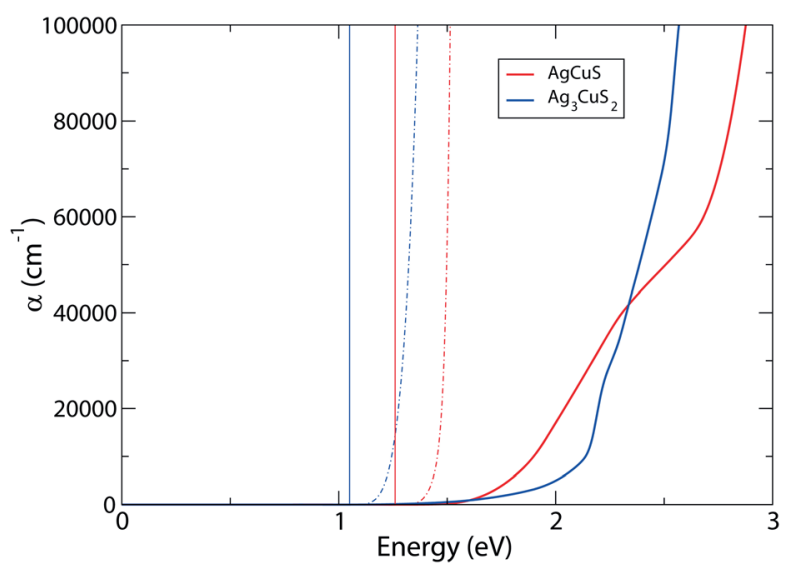

Fig. 7 Calculated optical absorption of $\mathrm{AgCuS}$ and $\mathrm{Ag}_{3} \mathrm{CuS}_{2}$, with: absorption coefficients marked as bold lines; fundamental band gaps marked by vertical lines; $(\alpha h \nu)^{2}$, representative of the predicted optical band gap, is marked as alternating dot-and-dash lines. transition in $\mathrm{Ag}_{3} \mathrm{CuS}_{2}$, as marked in Fig. $\mathrm{SF} 8, \uparrow$ is also dominated by $\mathrm{Cu}$ s states, unlike the $\mathrm{Cu} \mathrm{d}$ at the CBM.

To supplement this analysis, we have calculated the 'spectroscopic limited maximum efficiency' (SLME), a metric proposed by $\mathrm{Yu}$ and Zunger ${ }^{72,73}$ for assessing the theoretical maximum efficiency for both compounds, taking into account the nature of the band gap and the effect of the absorption, rather than the band gap alone. The SLME approach differs from the traditional SQ balance limit in two respects: first, it accounts for non-radiative recombination by treating the fraction of radiative electron-hole recombination current $\left(f_{\mathrm{r}}\right)$ as $f_{\mathrm{r}}=$ $\mathrm{e}^{-\Delta / k_{\mathrm{B}} T}$ where $k_{\mathrm{B}}$ is the Boltzmann constant, $T$, the temperature, and $\Delta$ is the difference between the lowest direct allowed transition and the fundamental band gap $\left(E_{\mathrm{g}}\right)$; as such, an absorber with higher $\Delta$ is expected to perform worse due to the greater non-radiative loss. Second, rather than taking the absorptivity, $a(E)$, as a step-wise function, with 0 below the band gap and 1 above, in SLME, it is a function of both absorption coefficient, $\alpha(E)$, and a thin film thickness, $L: a(E)=1-\mathrm{e}^{-2 \alpha(E) L}$. This additional assessment makes it particularly useful for screening potential photovoltaic materials, as it can identify materials that, while possessing an apparently suitable band gap according to the SQ limit, will be hampered by optical losses in real-world PV applications. Using a suitable film thickness of $2 \mu \mathrm{m}$, the SLME of AgCuS is $20.6 \%$, above the threshold $\mathrm{Yu}$ and Zunger indicated as 'high-SLME' (extrapolating to infinite thickness gives an absolute maximum of 27\%), while for $\mathrm{Ag}_{3} \mathrm{CuS}_{2}$, it is only $0.2 \%$. The low SLME of $\mathrm{Ag}_{3} \mathrm{CuS}_{2}$ is likely to occur due to a combination of a much larger $\Delta$ and a low absorption coefficient, both of which reduce efficiency. It is possible that the symmetry-forbidden transitions are made more likely at room temperature due to thermal effects, such as lattice expansion or defects disrupting the crystal symmetry, however the large number of these in $\mathrm{Ag}_{3} \mathrm{CuS}_{2}$ may cause major problems for its future application in solar cells, as indicated by the vast difference in SLME. This difference, compared with the similar theoretical efficiencies predicted by the SQ limit, highlights the need to move beyond the use of band gaps as the primary metric for screening potential PV materials.

\subsection{Electronic properties}

The charge transport properties of these compounds can be just as crucial to the construction of an efficient solar cell. To this end, the resistivity of $\mathrm{AgCuS}$ was measured; Hall effect measurements were also possible on the AgCuS pellet, allowing for the measurement of its carrier mobility. A comparison of these measurements with previously recorded values for champion third-generation absorbers CZTS and methylammonium lead iodide (MAPI) is shown in Table 2. The positive Hall coefficient of $\mathrm{AgCuS}$ is indicative of p-type conductivity, which agrees with the conductivity measurements of the orthorhombic phase performed by Guin et al. ${ }^{39}$

It was mentioned above that the effective masses of $\mathrm{AgCuS}$ are particularly low in some directions, close to those of CZTS; indeed, the mobility is the same magnitude as that of lower measurements of both CTZS and MAPI, which is as predicted, 
Table 2 Electronic behaviour of AgCuS, compared to other photovoltaic materials (stoichiometric, bar *, where S content ranges from 0-90\%, and $\mathrm{Cu}, \mathrm{Zn}$ and $\mathrm{Sn}$ ratios are variable)

\begin{tabular}{|c|c|c|c|c|}
\hline Material & Hall coefficient $\left(\mathrm{cm} \mathrm{C}^{-1}\right)$ & Bulk concentration $\left(\mathrm{cm}^{-3}\right)$ & Mobility $\left(\mathrm{cm}^{2} \mathrm{~V}^{-1} \mathrm{~s}^{-1}\right)$ & Resistivity $(\Omega \mathrm{cm})$ \\
\hline $\mathrm{AgCuS}$ & 0.2304 & $1.7 \times 10^{18}$ & 2.237 & 1.678 \\
\hline $\mathrm{CZTS}^{74}$ & - & $8 \times 10^{18}$ & 6.0 & 0.13 \\
\hline $\mathrm{CZT}(\mathrm{S}, \mathrm{Se}))^{* 76}$ & - & $1.73 \times 10^{16}$ to $1.4 \times 10^{15}$ & $0.46-1.32$ & $774-3300$ \\
\hline MAPI $^{77}$ & - & $\sim 10^{9}$ & 66 & $5.1 \times 10^{7}$ \\
\hline MAPI $^{78}$ & - & $2.8 \times 10^{17}$ & 3.9 & - \\
\hline
\end{tabular}

and very encouraging for its potential as an absorber layer in PV. In addition, as the pellet tested was pressed from a powder, and hence we might expect many grain boundaries and other defects, the measured mobility may be even higher for a stoichiometric thin film of AgCuS. Additional defects from our exploratory synthesis attempt may also be the cause of the relatively high carrier concentration in comparison to the device-quality values listed in Table 2; semiconductor-grade film growth may see this reduced as well. Previous work by Guin et al. calculated vacancy formation energies in $\mathrm{AgCuS}$ with $\mathrm{PBE}+\mathrm{U}$, finding $V_{\mathrm{Cu}}=+0.88 \mathrm{eV}$ per formula unit. ${ }^{39}$ This is comparable to $V_{\mathrm{Cu}}=+0.77 \mathrm{eV}$ found in $\mathrm{CZTS}^{20}$ and suggests that while such defects may be present at a reasonable concentration, their impact could be minimised with careful synthetic control. Antisite cation disorder of $\mathrm{Ag}$ and $\mathrm{Cu}$ in $\mathrm{AgCuS}$ is likely to be benign, however, as both cations are in the 1+ oxidation state. As such, a complete study of the defects in AgCuS at a high level of theory could be a worthwhile area for future study.

\subsection{Band alignment}

These results so far clearly indicate that $\mathrm{AgCuS}$ is a better candidate for photovoltaic applications than $\mathrm{Ag}_{3} \mathrm{CuS}_{2}$, with a more ideal band gap, stronger absorption onset and higher SLME. Thus, in order to aid in any further work towards AgCuS

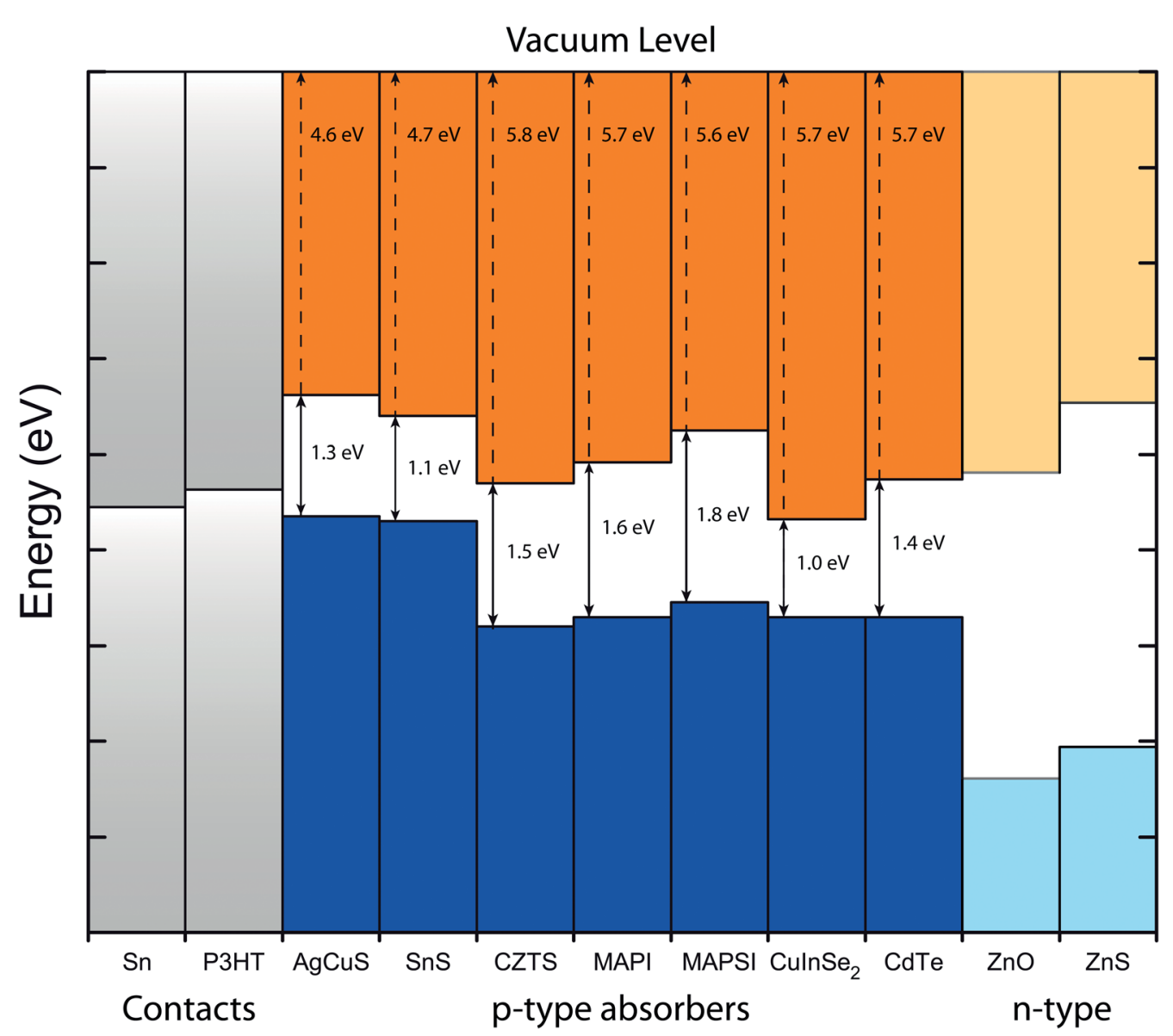

Fig. 8 Valence band alignment of AgCuS with a number of other materials. Ionisation potentials and workfunctions of other materials have been taken from the literature. ${ }^{28,79-84}$ 
as a photoabsorber, its valence band alignment with vacuum (ionisation potential) was calculated using the core-level alignment approach. ${ }^{57}$ This method has been used with other chalcogenide absorbers ${ }^{54}$ and MAPI, ${ }^{79}$ with particular success at assessing suitable hole-transporting and buffer layers for photovoltaic absorber materials. To this end, the alignment of AgCuS, in comparison with a number of other p-type solar absorber materials, plus some n-type and contact layers, is shown in Fig. 8.

Like SnS, it is clear that AgCuS has a much lower $(\sim 1 \mathrm{eV})$ predicted ionisation potential (IP) than other common photovoltaic absorber materials, including CZTS, which also has a valence band comprised primarily of $\mathrm{Cu} \mathrm{d}$ and $\mathrm{S} p$ states. One possible reason for this may be the unusual trigonal planar coordination of the copper atoms in the AgCuS structure, compared to tetrahedral coordination in kesterite. Indeed, structural distortion has been proposed as the reason for the IP of $\mathrm{SnS},{ }^{80}$ and coordination environment is known to have an effect on the local Madelung potential of atoms in crystals, ${ }^{85,86}$ resulting in changes in the VB position. ${ }^{\mathbf{1 1}}$ As a result of this difference in IP, the valence band level closely matches to the workfunctions of the organic conductor P3HT, and also Sn metal as buffer layers/contacts. On the other hand, $\mathrm{ZnO}$, as used in Liu et al.'s $\mathrm{Ag}_{3} \mathrm{CuS}_{2}$ cell, ${ }^{\mathbf{4 1}}$ has a large offset to the conduction band level of AgCuS, and we might expect would work poorly in a heterojunction cell with AgCuS. Instead, the related $\mathrm{ZnS}$ provides a much better match, with a conduction band level only $\sim 0.1 \mathrm{eV}$ below that of AgCuS. From these results, it may be anticipated that, for example, Sn/AgCuS/ZnS/FTO may show particular promise as a potential cell architecture in future work.

\section{Conclusion}

In this study, we have examined two of the silver copper sulfides experimentally and theoretically, in the context of photovoltaics. While both compounds, $\mathrm{AgCuS}$ and $\mathrm{Ag}_{3} \mathrm{CuS}_{2}$, are expected to demonstrate very conducive electronic structures for PV applications, including low carrier effective masses and suitable, direct band gaps, the optical behaviour and SLME of $\mathrm{Ag}_{3} \mathrm{CuS}_{2}$ indicates that it will be severely limited by optical losses when used as an absorber. AgCuS possesses a more ideal band gap of $1.25 \mathrm{eV}$, observed theoretically and experimentally, good carrier mobilities and is predicted to exhibit a higher SLME and stronger optical absorption, and so it is anticipated to be a much more viable candidate for further study into the use of these materials in photovoltaics.

\section{Acknowledgements}

The work in this article used the UCL Legion HPC Facility (Legion@UCL), the Iridis cluster, provided by the EPSRC funded Centre for Innovation (Grant codes EP/K000144/1 and EP/ K000136/1) and the ARCHER UK National Supercomputing Service. Access to ARCHER was provided through the UK's HEC Materials Chemistry Consortium, funded by EPSRC (EP/ L000202). CNS is grateful to the Department of Chemistry at
UCL for the provision of a DTA studentship, and AMG acknowledges Diamond Light Source for the co-sponsorship of a studentship on the EPSRC Centre for Doctoral Training in Molecular Modelling and Materials Science (EP/L015862/1). DOS and RGP acknowledge support from the SUPERSOLAR Solar Energy Hub (EP/J017361/1) for the provision of a flexible funding call award, and membership of the Materials Design Network.

\section{References}

1 A. Jäger-Waldau, Sol. Energy Mater. Sol. Cells, 2012, 95, 373395.

2 L. M. Peter, Philos. Trans. R. Soc. London, Ser. A, 2011, 369, 1840-1856.

3 J. Peng, L. Lu and H. Yang, Renewable Sustainable Energy Rev., 2013, 19, 255-274.

4 M. A. Green, K. Emery, Y. Hishikawa, W. Warta and E. D. Dunlop, Progress in Photovoltaics: Research and Applications, 2015, vol. 23, pp. 805-812.

5 W. Shockley and H. J. Queisser, J. Appl. Phys., 1961, 32, 510519.

6 A. B. Kehoe, D. J. Temple, G. W. Watson and D. O. Scanlon, Phys. Chem. Chem. Phys., 2013, 15, 15477-15484.

7 M. Kumar and C. Persson, J. Renewable Sustainable Energy, 2013, 5, 031616.

8 D. J. Temple, A. B. Kehoe, J. P. Allen, G. W. Watson and D. O. Scanlon, J. Phys. Chem. C, 2012, 116, 7334-7340.

9 D. O. Scanlon and A. Walsh, Appl. Phys. Lett., 2012, 100, 251911.

10 A. N. Fioretti, A. Zakutayev, H. Moutinho, C. Melamed, J. D. Perkins, A. G. Norman, M. Al-Jassim, E. S. Toberer and A. C. Tamboli, J. Mater. Chem. C, 2015, 3, 11017-11028.

11 T. D. Veal, N. Feldberg, N. F. Quackenbush, W. M. Linhart, D. O. Scanlon, L. F. J. Piper and S. M. Durbin, Adv. Energy Mater., 2015, 5, 1501462.

12 T. Yokoyama, F. Oba, A. Seko, H. Hayashi, Y. Nose and I. Tanaka, Appl. Phys. Express, 2013, 6, 061201.

13 Y. Zhou, L. Wang, S. Chen, S. Qin, X. Liu, J. Chen, D.-J. Xue, M. Luo, Y. Cao, Y. Cheng, E. H. Sargent and J. Tang, Nat. Photonics, 2015, 9, 409-415.

14 R. X. Yang, K. T. Butler and A. Walsh, J. Phys. Chem. Lett., 2015, 6, 5009-5014.

15 A. W. Welch, L. L. Baranowski, P. Zawadzki, S. Lany, C. A. Wolden and A. Zakutayev, Appl. Phys. Express, 2015, 8, 082301.

16 B. Yang, L. Wang, J. Han, Y. Zhou, H. Song, S. Chen, J. Zhong, L. Lv, D. Niu and J. Tang, Chem. Mater., 2014, 26, 3135-3143.

17 Y. C. Choi, E. J. Yeom, T. K. Ahn and S. I. Seok, Angew. Chem., Int. Ed., 2015, 54, 4005-4009.

18 Y. C. Choi, D. U. Lee, J. H. Noh, E. K. Kim and S. I. Seok, Adv. Funct. Mater., 2014, 24, 3587-3592.

19 W. Wang, M. T. Winkler, O. Gunawan, T. Gokmen, T. K. Todorov, Y. Zhu and D. B. Mitzi, Adv. Energy Mater., 2014, 4, 1301465. 
20 S. Chen, J.-H. Yang, X. G. Gong, A. Walsh and S.-H. Wei, Phys. Rev. B: Condens. Matter Mater. Phys., 2010, 81, 245204.

21 G. Larramona, S. Bourdais, A. Jacob, C. Choné, T. Muto, Y. Cuccaro, B. Delatouche, C. Moisan, D. Péré and G. Dennler, J. Phys. Chem. Lett., 2014, 5, 3763-3767.

22 S. Siebentritt, Thin Solid Films, 2013, 535, 1-4.

23 T. Gershon, T. Gokmen, O. Gunawan, R. Haight, S. Guha and B. Shin, MRS Commun., 2014, 4, 159-170.

24 S. Bourdais, C. Choné, B. Delatouche, A. Jacob, G. Larramona, C. Moisan, A. Lafond, F. Donatini, G. Rey, S. Siebentritt, A. Walsh and G. Dennler, Adv. Energy Mater., 2016, 6, 1502276.

25 S. Chen, A. Walsh, J.-H. Yang, X. G. Gong, L. Sun, P.-X. Yang, J.-H. Chu and S.-H. Wei, Phys. Rev. B: Condens. Matter Mater. Phys., 2011, 83, 125201.

26 S. Chen, A. Walsh, X.-G. Gong and S.-H. Wei, Adv. Mater., 2013, 25, 1522-1539.

27 Y. S. Lee, T. Gershon, O. Gunawan, T. K. Todorov, T. Gokmen, Y. Virgus and S. Guha, Adv. Energy Mater., 2015, 5, 1401372.

28 A. Walsh, S. Chen, S.-H. Wei and X.-G. Gong, Adv. Energy Mater., 2012, 2, 400-409.

29 T. Gershon, Y. S. Lee, R. Mankad, O. Gunawan, T. Gokmen, D. Bishop, B. McCandless and S. Guha, Appl. Phys. Lett., 2015, 106, 123905.

30 T. Gershon, B. Shin, N. Bojarczuk, M. Hopstaken, D. B. Mitzi and S. Guha, Adv. Energy Mater., 2015, 5, 1400849.

31 S. Djurle, Acta Chem. Scand., 1958, 12, 1427-1436.

32 R. F. Kadrgulov, R. A. Yakshibaev and M. A. Khasanov, Ionics, 2001, 7, 156-160.

33 C. L. Baker, F. J. Lincoln and A. W. S. Johnson, Acta Crystallogr., Sect. B: Struct. Sci., 1991, 47, 891-899.

34 C. Baker, F. Lincoln and A. Johnson, Aust. J. Chem., 1992, 45, 1441-1449.

35 D. M. Trots, A. Senyshyn, D. A. Mikhailova, M. Knapp, C. Baehtz, M. Hoelzel and H. Fuess, J. Phys.: Condens. Matter, 2007, 19, 136204.

36 D. M. Trots, A. Senyshyn, D. A. Mikhailova, T. Vad and H. Fuess, J. Phys.: Condens. Matter, 2008, 20, 455204.

37 A. D. Davletshina, R. A. Yakshibaev, N. N. Bikkulova, Y. M. Stepanov and L. V. Bikkulova, Solid State Ionics, 2014, 257, 29-31.

38 D. Santamaria-Perez, A. Morales-Garcia, D. Martinez-Garcia, B. Garcia-Domene, C. Mühle and M. Jansen, Inorg. Chem., 2013, 52, 355-361.

39 S. N. Guin, J. Pan, A. Bhowmik, D. Sanyal, U. V. Waghmare and K. Biswas, J. Am. Chem. Soc., 2014, 136, 12712-12720.

40 C. Xing, Y. Zhang, Z. Wu, D. Jiang and M. Chen, Dalton Trans., 2014, 43, 2772-2780.

41 Z. Liu, J. Han, K. Guo, X. Zhang and T. Hong, Chem. Commun., 2015, 51, 2597-2600.

42 Y. Lei, X. Yang, L. Gu, H. Jia, S. Ge, P. Xiao, X. Fan and Z. Zheng, J. Power Sources, 2015, 280, 313-319.

43 D. O. Scanlon and A. Walsh, Acta Crystallogr., Sect. B: Struct. Sci., Cryst. Eng. Mater., 2015, 71, 702-706.

44 G. Kresse and J. Hafner, Phys. Rev. B: Condens. Matter Mater. Phys., 1993, 47, 558-561.
45 G. Kresse and J. Hafner, Phys. Rev. B: Condens. Matter Mater. Phys., 1994, 49, 14251-14269.

46 G. Kresse and J. Furthmüller, Phys. Rev. B: Condens. Matter Mater. Phys., 1996, 54, 11169-11186.

47 G. Kresse and J. Furthmüller, Comput. Mater. Sci., 1996, 6, 15. 48 A. V. Krukau, O. A. Vydrov, A. F. Izmaylov and G. E. Scuseria,

J. Chem. Phys., 2006, 125, 224106.

49 J. Perdew, K. Burke and M. Ernzerhof, Phys. Rev. Lett., 1996, 77, 3865-3868.

50 P. Blochl, Phys. Rev. B: Condens. Matter Mater. Phys., 1994, 50, 17953-17979.

51 J. P. Perdew and M. Levy, Phys. Rev. Lett., 1983, 51, 18841887.

52 B. G. Janesko, T. M. Henderson and G. E. Scuseria, Phys. Chem. Chem. Phys., 2009, 11, 443-454.

53 A. B. Kehoe, D. O. Scanlon and G. W. Watson, Phys. Rev. B: Condens. Matter Mater. Phys., 2011, 83, 233202.

54 A. M. Ganose, K. T. Butler, A. Walsh and D. O. Scanlon, J. Mater. Chem. A, 2016, 4, 2060-2068.

55 C. N. Savory, R. G. Palgrave, H. Bronstein and D. O. Scanlon, Sci. Rep., 2016, 6, 20626.

56 M. Gajdoš, K. Hummer, G. Kresse, J. Furthmüller and F. Bechstedt, Phys. Rev. B: Condens. Matter Mater. Phys., 2006, 73, 045112.

57 S.-H. Wei and A. Zunger, Appl. Phys. Lett., 1998, 72, 20112013.

58 K. Momma and F. Izumi, J. Appl. Crystallogr., 2011, 44, 12721276.

59 Y. Tokuhara, K. Tezuka, Y. J. Shan and H. Imoto, J. Ceram. Soc. Jpn., 2009, 117, 359-362.

60 D. O. Scanlon and G. W. Watson, J. Phys. Chem. Lett., 2010, 1, 2582-2585.

61 J. P. Allen, D. O. Scanlon and G. W. Watson, Phys. Rev. B: Condens. Matter Mater. Phys., 2011, 84, 115141.

62 G. Hautier, A. Miglio, D. Waroquiers, G.-M. Rignanese and X. Gonze, Chem. Mater., 2014, 26, 5447-5458.

63 H.-R. Liu, S. Chen, Y.-T. Zhai, H. J. Xiang, X. G. Gong and S.-H. Wei, J. Appl. Phys., 2012, 112, 093717.

64 J. M. Frost, K. T. Butler, F. Brivio, C. H. Hendon, M. van Schilfgaarde and A. Walsh, Nano Lett., 2014, 14, 25842590.

65 G. Giorgi, J.-I. Fujisawa, H. Segawa and K. Yamashita, J. Phys. Chem. Lett., 2013, 4, 4213-4216.

66 C. Persson, J. Appl. Phys., 2010, 107, 053710.

67 S. N. Guin, D. Sanyal and K. Biswas, Chem. Sci., 2016, 7, 534543.

68 B. Chowdari, K. Mok, J. Xie and R. Gopalakrishnan, J. NonCryst. Solids, 1993, 160, 73-81.

69 J. Gebhardt, J. McCarron, P. Richardson and A. Buckley, Hydrometallurgy, 1986, 17, 27-38.

70 D. L. Perry and J. A. Taylor, J. Mater. Sci. Lett., 1986, 5, 384386.

71 J. Yeh and I. Lindau, At. Data Nucl. Data Tables, 1985, 32, 1155.

72 L. Yu and A. Zunger, Phys. Rev. Lett., 2012, 108, 068701.

73 L. Yu, R. S. Kokenyesi, D. A. Keszler and A. Zunger, Adv. Energy Mater., 2013, 3, 43-48. 
74 T. Tanaka, T. Nagatomo, D. Kawasaki, M. Nishio, Q. Guo, A. Wakahara, A. Yoshida and H. Ogawa, J. Phys. Chem. Solids, 2005, 66, 1978-1981.

75 F. Liu, Y. Li, K. Zhang, B. Wang, C. Yan, Y. Lai, Z. Zhang, J. Li and Y. Liu, Sol. Energy Mater. Sol. Cells, 2010, 94, 2431-2434.

76 K. F. Tai, O. Gunawan, M. Kuwahara, S. Chen, S. G. Mhaisalkar, C. H. A. Huan and D. B. Mitzi, Adv. Energy Mater., 2016, 6, 1501609.

77 C. C. Stoumpos, C. D. Malliakas and M. G. Kanatzidis, Inorg. Chem., 2013, 52, 9019-9038.

78 Q. Wang, Y. Shao, H. Xie, L. Lyu, X. Liu, Y. Gao and J. Huang, Appl. Phys. Lett., 2014, 105, 163508.

79 K. T. Butler, J. M. Frost and A. Walsh, Mater. Horiz., 2015, 2, 228-231.

80 L. A. Burton and A. Walsh, Appl. Phys. Lett., 2013, 102, 132111.
81 A. M. Ganose, C. N. Savory and D. O. Scanlon, J. Phys. Chem. Lett., 2015, 6, 4594-4598.

82 R. K. Swank, Phys. Rev., 1967, 153, 844-849.

83 G. Teeter, J. Appl. Phys., 2007, 102, 034504.

84 R. J. Davis, M. T. Lloyd, S. R. Ferreira, M. J. Bruzek, S. E. Watkins, L. Lindell, P. Sehati, M. Fahlman, J. E. Anthony and J. W. P. Hsu, J. Mater. Chem., 2011, 21, 1721-1729.

85 D. O. Scanlon, C. W. Dunnill, J. Buckeridge, S. A. Shevlin, A. J. Logsdail, S. M. Woodley, C. R. A. Catlow, M. J. Powell, R. G. Palgrave, I. P. Parkin, G. W. Watson, T. W. Keal, P. Sherwood, A. Walsh and A. A. Sokol, Nat. Mater., 2013, 12, 798-801.

86 J. Buckeridge, K. T. Butler, C. R. A. Catlow, A. J. Logsdail, D. O. Scanlon, S. A. Shevlin, S. M. Woodley, A. A. Sokol and A. Walsh, Chem. Mater., 2015, 27, 3844-3851. 